Pub. Mat. UAB

vol. 29 No 2-3 liov. 1985

\title{
CENTRAL EXTENSION AND COVERINGS
}

zdzislaw Wojtkowiak*

The theory of central extensions has a lot of analogy with

the theory of covering spaces. It is mentioned for example in [1]. In this paper we show that the category of central extensions of a perfect group and a certain category of covering spaces of a certain space are equivalent (see Theoren 1). Then the facts about central extensions will follow from the corresponding facts about coverings (see corollaries $x-3$ ).

We start with some definitions to make this work selfcontained.

Definition ]. (see [2]\&5) A pair (X; $)$ is called a central extension of a group $G$ if $\varepsilon: X \rightarrow G$ is an epimorphism and kernel(c) center $X$ Definition 2 . (see $[2] \$ 5$ ) The central extension $(X ; 0)$ of a group $G$ is called universal if for every central extension $(Y ; \omega)$ of $G$ there is one and only one homomorphism $h: X \rightarrow Y$ such that $\Psi$ oh $=\mathbb{E}$. It follows from [2] Theorem 5.3 that if a group $G$ has universal central extension $(x: c)$ then $G$ and $x$ are perfect.

We shall denote by $E(G)$ the category of central extensions of $G$. Morphisms in this category are homomorphisms over $G$.

Now we describe a category $\operatorname{cov}^{a b}(x)$ of pointed abelian coverings over a connecteo space $x$ with a base point. Objects of cov. ${ }^{a b}(x)$ are principal G-fibrations over $X$ with a base point in the fibre over the base point of $X$. $G$ is a discrete abelian group. Such principal G-fibrations are regular coverings and they are inouced from the universal covering of $B G$ by a map $f: X \rightarrow B G$. If $E_{1}$ and $E_{2}$ are

* Supported by Forschungsinstitut für Mathematik, ETH-Zentrum, CH-8092 Zürich 
two coverings inouced respectively by $f_{1}: x \rightarrow B G_{1}$ and $f_{2}: x \rightarrow B G_{2}$ then morphisms of $E_{1}$ in $E_{2}$ in the category. cov. ${ }^{a b}(x)$ are those pointed maps from. $E_{1}$ in $E_{2}$ over $X$ which are induced by maps $h: B G_{1} \rightarrow B G_{2}$ such that $h_{0} f_{1}$ is homotopic to $f_{2}$. The category cov. ${ }^{a b}(x)$ has an initial object. It is the universal, pointed covering.

Let us suppose now that $G$ is a perfect group. Then the fundamental group of $B G$ is perfect and we can apply the "f" construction to get $\mathrm{BG}^{+} . \mathrm{BG}^{+}$is simply-connected and therefore $\Omega\left(\mathrm{BG}^{+}\right)$is connected.

Theorem 1 . Let $G$ be a perfect group. Then the categories cov. ${ }^{\mathrm{ab}}\left(\Omega\left(\mathrm{BG}^{+}\right)\right)$ ano $E(G)$ are equivalent. The full subcategory of $\operatorname{cov}^{a b}\left(\Omega\left(B G^{+}\right)\right)$which objects are connected coverings and the category of central extensions $(X, p)$ of $G$ such that $X^{\prime}$ s are perfect,are also equivalent.

Proof. We shall define two functors $F: E(G) \rightarrow \operatorname{Cov}^{a b}\left(\Omega \mathrm{BG}^{+}\right)$and $J=\operatorname{cov}^{a b}\left(\Omega \mathrm{BG}^{+}\right) \rightarrow E(G)$ such that the compositions $F_{0} J$ and JoF are natural isomorphic to the identity functors.

Let $1 \rightarrow \mathrm{H} \rightarrow \mathrm{X} \stackrel{\varphi}{\rightarrow} \mathrm{G} \rightarrow I$ be a central extension. Then $\mathrm{BH} \rightarrow \mathrm{BX} \rightarrow \mathrm{BG}$ is a fibration. Let $\mathrm{tr}: \mathrm{H}_{2}(\mathrm{BG})-\mathrm{H}_{1}(\mathrm{BH})$ be a transgression homomorphism in the serre spectral sequence of this fibration. The homomorphism $t r$ we can consider as an element $t \in \mathrm{H}^{2}$ (BG,H) $=$ $\mathrm{H}^{2}\left(\mathrm{BG}^{+} \mathrm{H}\right)$. We have the following long sequence of fibrations $\left\langle{ }^{*} \mathrm{X}^{\prime} \rightarrow \Omega \delta(\mathrm{X}) \rightarrow \Omega \mathrm{BG}^{+} \stackrel{\delta=\Omega \mathrm{t}}{\longrightarrow} \mathrm{K}(\mathrm{H}, 1) \rightarrow \delta(\mathrm{X}) \rightarrow \mathrm{BG}^{+} \stackrel{t}{\rightarrow} \mathrm{K}(\mathrm{H}, 2)\right.$ where $F(x)$ is a homotopy fibre of $t$. We set $F(X: \varphi)=\left(\delta !(E H) \rightarrow \Omega\left(\mathrm{BG}^{+}\right)\right)$where $\delta !(\mathrm{EH}) \rightarrow \Omega\left(\mathrm{BG}^{+}\right)$is a 
covering induced by of from the universal covering over BH. The base point of $\delta$ !EH we choose in the fibre over the base point of $\Omega\left(B^{+}\right)$. The homomorphism $f:\left(x_{1}, \varphi_{1}\right) \rightarrow\left(X_{2}, \varphi_{2}\right)$ of central extensions induces a map between sequences of fibrations $\left({ }^{\star} \mathrm{x}_{1}\right)$ and $\left({ }^{*} \mathrm{x}_{2}\right)$. As a part of this map we get a commutative diagram

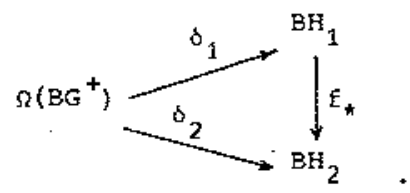

This diagram induces a morphism between coverings $\delta_{1} !\left(\mathrm{EH}_{1}\right) \rightarrow{ }_{\Omega}\left(\mathrm{BG}^{+}\right)$ and $\partial_{2}:\left(\mathrm{EH}_{2}\right)-\Omega\left(\mathrm{BG}^{+}\right)$in the category $\mathrm{Cov} \cdot{ }^{\mathrm{ab}}\left(\Omega\left(\mathrm{BG}^{+}\right)\right)$.

Now we shall define a functor $J: \operatorname{Cov}^{\mathrm{ab}}\left(\Omega \mathrm{BG}^{+}\right) \rightarrow \mathrm{E}(\mathrm{G})$. Let

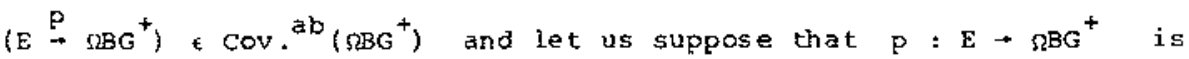
a principal $\mathrm{K}$ fibration. ( $\mathrm{P}: \mathrm{E} \rightarrow \Omega \mathrm{BG}^{+}$) is induced from the universal covering over $B K$ by a map $x: \Omega\left(B G^{+}\right) \rightarrow B K$. We have the following isomorphisms

$\mathrm{H}^{1}\left(\Omega\left(\mathrm{BG}^{+}\right) ; \mathrm{K}\right) \approx \operatorname{Hom}\left(\pi_{1}\left(\Omega \mathrm{BG}^{+}\right) ; \mathrm{K}\right) \approx \operatorname{Hom}\left(\pi_{2}\left(\mathrm{BG}^{+}\right) ; \mathrm{K}\right) \approx \mathrm{H}^{2}\left(\mathrm{BG}^{+} ; \mathrm{K}\right)$. Therefore there is $y \in \mathrm{H}^{2}\left(\mathrm{BG}^{+} ; \mathrm{K}\right)$ which corresponds to $\mathrm{x}$ by these isomorphisms. Let us form the following sequence of fibrations

$$
(\star \star) \quad \rightarrow \Omega B G^{+} \stackrel{\Omega Y=x}{\longrightarrow} \mathrm{K}(\mathrm{H} ; 1) \rightarrow \mathrm{Y}=\text { Fibre }(\mathrm{Y}) \rightarrow \mathrm{BG}^{+} \stackrel{\mathrm{Y}}{\longrightarrow} \mathrm{K}(\mathrm{H} ; 2) .
$$

Let $\mathrm{i}: \mathrm{BG}-\mathrm{BG}^{+}$be a natural map in the " $+"$ construction. Let

$$
\{* * *) \quad \mathrm{K}(\mathrm{H} ; 1) \rightarrow \mathrm{S}=\mathrm{i}: \mathrm{Y} \rightarrow \mathrm{BG}
$$

be a fibration induced by $i$ from the fibration

$$
\mathrm{K}(\mathrm{H}: 1) \rightarrow \mathrm{Y} \rightarrow \mathrm{BG}^{+} \text {. }
$$


After applying functor $\pi_{1}$ to the fibration (***) we get an exact sequence

$(* \star \star \star) \quad 1 \rightarrow \mathrm{H} \rightarrow \pi_{1}(\mathrm{~S})=\mathrm{T} \rightarrow \mathrm{G} \rightarrow 1$.

The action of $\pi_{1}(\mathrm{BG})$ on the fibre in the fibration (***) is trivial because this fibration is induced from the fibration over the

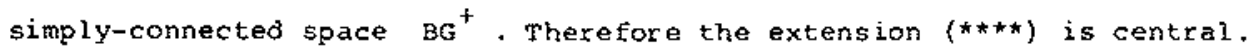

A map in the category $\operatorname{cov} \cdot{ }^{a b}\left(\Omega B G^{+}\right)$induces a homotopy conmutative diagram

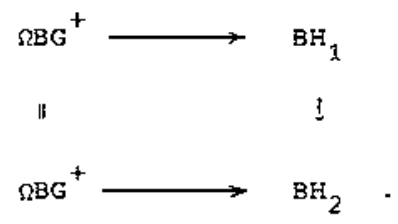

Hence we get a homotopy commutative diagram

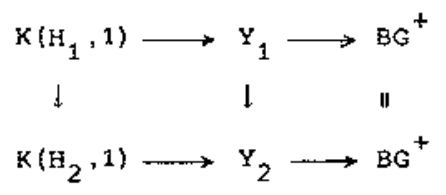

and consequently a map between central extensions

$$
\begin{array}{ccccc}
H_{1} & -T_{1} & \rightarrow & G \\
\downarrow & \downarrow & & \\
H_{2} & \rightarrow & T_{2} & \rightarrow & G
\end{array}
$$

The proof that the compositions $F_{0} J$ and $J_{0} F$ are natural isomorphic to the identities follows immediately from definitions of $F$ and $J$ and $I$ omit it.

If a principal H-fibration $\mathrm{E} \rightarrow \Omega \mathrm{BG}^{+}$is connected then $\pi_{1}\left(S B G^{+}\right) \rightarrow \pi_{1}(B H)$ is an epimorphism.Thisimplies that $\pi_{1}(Y)=0$ and 
therefore $H_{1}(T)=0$. Consequently $J\left(E \rightarrow \Omega B G^{+}\right)=(1 \rightarrow H \rightarrow T+G+1)$ is an extension of $G$ such that $T$ is perfect.

If $1 \rightarrow \mathrm{H} \rightarrow \mathrm{X} \rightarrow \mathrm{G} \rightarrow 1$ is a central extension with $\mathrm{X}$ perfect then $\pi_{1}\left(\Omega \mathrm{BG}^{+}\right) \rightarrow \pi_{1}(\mathrm{BH})$ is an epimorphism and consequentiy the induced covering over $\Omega B G^{+}$is connected.

The following corollaries, usually proved in an algebraic way, follow immediately from Theorem 1 .

Corollary 1. There exists a universal central extension of a perfect group G.

Proof. The universal central extension is an initial object in the category $E(G)$. The category cov. ${ }^{2 b}\left(\Omega B G^{+}\right)$has an initial object. It is a universal covering. Therefore there is an initial object in $E(G)$. Corollary 2. $(X: \varphi)$ is a universal extension iff $H_{1}(X)=0$ ano $\mathrm{H}_{2}(\mathrm{X})=0$. Then we have ker $\varphi=\mathrm{H}_{2}(G)$.

Proof. The principal fibration corresponding to $(X ; \varphi)$ is $\Omega \mathrm{BX}^{+} \rightarrow \mathrm{\Omega BG}^{+}$. This covering is universal if and only if $\pi_{0}\left(\Omega \mathrm{BX}^{+}\right)=0$ and $\pi_{1}\left(\Omega B \mathrm{X}^{+}\right)=0$. Hence we have that $(X ; \varphi)$ is universal if and only if $\mathrm{H}_{1}\left(\mathrm{BX}^{+}\right)=\mathrm{H}_{1}(\mathrm{X})=0$ and $\mathrm{H}_{2}\left(\mathrm{BX}^{+}\right)=\mathrm{H}_{2}(\mathrm{X})=0$. The fibration $\Omega \mathrm{BX}^{+} \rightarrow \Omega \mathrm{BG}^{+}$is induced from the universal covering over $\mathrm{B}(\mathrm{ker} \varphi)$ by a map $\Omega \mathrm{BG}^{+} \rightarrow \mathrm{B}(\operatorname{ker} \varphi)$. If it is universal then $\operatorname{ker} \varphi=\pi_{1}\left(\Omega \mathrm{BG}^{+}\right)=\pi_{2}\left(\mathrm{BG}^{+}\right)=\mathrm{H}_{2}\left(\mathrm{BG}^{+}\right)=\mathrm{H}_{2}(\mathrm{G})$.

Corollary 3 . The isomorphism classes of central extensions $(X, \varphi)$ of $G$ such that $X$ 's are perfect, are in one to one correspondence with subsroups of $\mathrm{H}_{2}(\mathrm{G})$.

Proof. The isomorphism classes of connected coverings over $\Omega \mathrm{BG}^{+}$are in one to one correspondence with subgroups of $\pi_{1}\left(\Omega B G^{+}\right)=H_{2}(G)$. 
Sone steps in the proofs given below can be shown using the following proposition which itself seems to be interesting .

Proposition 1 . Let us suppose that $O \rightarrow H \rightarrow X \rightarrow G \rightarrow 1$ is a central extension of a perfect group $G$ by a group $H$. Then $\mathrm{BH} \rightarrow \mathrm{BX}^{+} \rightarrow \mathrm{BG}^{+}$is a fibration. TThe "+" construction is ane with respect to a maxinal perfect subgroup of $\mathrm{x}$.)

Proof. Let us assume first that $x$ is perfect. Let $F$ be a fibre of $\mathrm{BX}^{+} \rightarrow \mathrm{BG}^{+}$. There is a map of a fibration $\mathrm{BH} \rightarrow \mathrm{BX} \rightarrow \mathrm{BG}$ into a fibration $\mathrm{F} \rightarrow \mathrm{BX}^{+} \rightarrow \mathrm{BG}^{+}$. This map induces a map of serre spectral sequences. This map is an isomorphism on $E_{\star, 0}^{2}$ and on $E_{\star \star \star}^{\infty}$-terms. Therefore it is isomorphism on $E_{O_{1}}^{2}$-terms. This means that a map $\mathrm{H}_{\star}(\mathrm{BH} ; \mathrm{Z})-\mathrm{H}_{\star}(F ; Z)$ is an isomorphism. $\mathrm{F}$ is a fibre of a map between: nilpotent spaces therefore it is nilpotent. It implies that $B H \rightarrow F$ is a homotopy equivalence.

Let now $x$ be arbitrary and lat $x^{*}$ be a maximal, perfect subgroup of $X$. The extension $O \rightarrow H^{*}=\operatorname{Ker}(i) \rightarrow X^{\prime} \stackrel{i}{\rightarrow} G \rightarrow 1$ is also central. Horeover $\mathrm{BX}^{+}$is a universal cover of $\mathrm{BX}^{+}$. If $F$ is a fibre of $\mathrm{BX}^{+} \rightarrow \mathrm{BG}^{+}$then only $\pi_{1}(F)$ is non-zexo and it appears in the following exact sequence

$$
O \rightarrow \pi_{2}\left(\mathrm{BG}^{+}\right) \rightarrow \pi_{1}(\mathrm{~F}) \rightarrow \pi_{1}\left(\mathrm{BX}^{+}\right) \rightarrow 1
$$

$\pi_{1}\left(B x^{+}\right)$is abelian. This implies that $\pi_{1}(F)$ is nilpotent. Repeating once more arguments with the Serre spectral seguence we get that $F$ is homotopically equivalent to $\mathrm{K}(\mathrm{H}, 1)$.

In [3] we have introduceo $"{ }_{P}{ }^{17}$ construction in the case if $H_{1}\left(X ; z_{P}\right)=0 .\left(z_{P}\right.$ is a ring of integers localized outside $P$.) 
Definition 3 . We say that $G$ is p-perfect if $H_{1}\left(G, z_{p}\right)=0$.

We shall study central extensions of a P-perfect group $G$ by finitely generated $z_{p}$-modules. We shall denote this category by $E_{p}(G)$. We have the following proposition.

Proposition 2. Let $\mathrm{O} \rightarrow \mathrm{H} \rightarrow \mathrm{X} \rightarrow \mathrm{G} \rightarrow 1$ be a central extension of $\mathrm{a}$ P-perfect group $G$ by a finitely generated $z_{p}$-module $H$. Then $\mathrm{BH} \rightarrow \mathrm{BX}^{+} \mathrm{P} \rightarrow \mathrm{BG}^{+} \mathrm{P}$ is a fibration. (The "+ $"$-construction is one with respect to a maximal p-perfect subgroup of $x$ ).

The proof of Proposition 2 is exactly the same as the proof of Proposition 1 . .

Let $X$ be a P-local space. We define a category cov ${ }_{, P}^{a b}(x)$. objects of cov. ${ }_{, p}(x)$ are principal M-fibrations over $x$ with a fixed base point in the fibre over the base point of $x$. M is a $z_{p}$-mooule.

Theorem 2. Let $G$ be a P-perfect group. Then the categories $\operatorname{Cov}_{p}^{a b}\left(\Omega B G^{+} P\right)$ and $E_{p}(G)$ are equivalent. The full subcategory of cov. ${ }_{, P}^{a b}\left(\Omega B{ }^{+} P\right)$ which objects are connected coverings and the category of central extensions $(X, \varphi)$ of $G$ such that $X^{\prime} s$ are $P$-perfect, are also equivalent.

Corollaxy 4. i) There exists a universal central extension of a p-perfect group $G$ in the category $\varepsilon_{P}(G)$.

ii) $(X, \varphi)$ is a universal central extension of a P-perfect group $G$ in the category $E_{p}(G)$ if and only if $H_{1}\left(x ; z_{p}\right)=0$ and $H_{2}\left(x ; z_{p}\right)=0$. Then we have that ker $\varphi=H_{2}\left(G ; Z_{p}\right)$.

iii) The isomorphisn classes of central extensions of $G$ by p-perfect groups in the category $E_{p}(G)$ are in one to one correspondence with 
$\mathrm{z}_{\mathrm{p}}$-submodules of $\mathrm{H}_{2}\left(\mathrm{G}, \mathrm{z}_{\mathrm{P}}\right)$.

The proofs are the same as before.

Proposition 3 . Let $H \rightarrow X+G$ be a central extension of $G$. Then there is a central extension of $G$ by $H \otimes z_{p}$ together with a natural map

$$
\begin{aligned}
& \mathrm{O} \rightarrow \mathrm{H} \rightarrow \mathrm{X} \rightarrow \mathrm{G} \rightarrow 1 \\
& \text { i } \downarrow \text { ॥ } \\
& 0 \rightarrow \operatorname{HgZ}_{p} \rightarrow \mathrm{x}_{\mathrm{p}} \rightarrow \mathrm{G} \rightarrow 1
\end{aligned}
$$

where $i$ is $z_{p}$-localization $(i(a)=a \otimes 1)$.

Eroof. We have a Fibration

(*) $\mathrm{BH} \rightarrow \mathrm{BX} \rightarrow \mathrm{BG}$.

Bousfielo and Kan have introduced the fibrewise localization functor. After applying it to a fibration (*) we obtain a fibration

$(\star \star)\langle\mathrm{BH})_{\mathrm{P}} \rightarrow(\mathrm{BX})_{\mathrm{P}}^{+} \rightarrow \mathrm{BG}$

and a fibre map of $(*)$ into $\left({ }^{\star}\right)$.

From the fibration (**) we get the following exact sequence

$$
0 \rightarrow H \otimes z_{p} \rightarrow \pi_{1}\left(B x_{p}^{f}\right):=x_{P} \rightarrow G \rightarrow 1 \text {. }
$$

The action of $\pi_{1}(\mathrm{BG})=G$ on fibres of $(*)$ and $(* *)$ are compatible therefore (**)is a central extension.

Proposition 3 is of course a special case of a more general result proved by algebraic method in [0]. Proposition 1 is of course well. known. The related results about "t" construction are also in A.J. Berrick "An Approach to Algebraic $x$-theory", Pitman research notes in Math. 56 (London, 1982).

References

[0] P.Hilton, Relative nilpotent groups, Lecture Notes in Math. 915, Springer-Verlag 1982 . 
[1] M.A. Kervaire, Multiplicateurs de Schur et K-théorie, in Essays on Topology and Related Topics, Springer-Verlag 1970.

[2] J. Milnor, Introduction to algebraic K-theory, Princeton University press, 1971.

[3] z. Wojtkowiak, On fibrations which are also cofibrations, Quart. J. Math. Oxford (2), 30 (1979), 505-512.

Rebux el is de mars del $\$ 985$

Universitat Autònoma de Barcelona

Facultat de Ciencies

Departament de Matematiques

Bellaterra - Barcelona

SPAIN 